\title{
Paraxial WKB method applied to the lower hybrid wave propagation ${ }^{\text {a) }}$
}

\author{
N. Bertelli, ${ }^{1, b)}$ O. Maj, ${ }^{2}$ E. Polii, ${ }^{2}$ R. Harvey, ${ }^{3}$ J. C. Wright, ${ }^{4}$ P. T. Bonoli, ${ }^{4}$ C. K. Phillips, ${ }^{1}$ E. Valeo, ${ }^{1}$ and \\ J. R. Wilson ${ }^{1}$ \\ 1) Princeton Plasma Physics Laboratory, Princeton, NJ 08543, USA \\ ${ }^{2)}$ Max-Planck-Institut für Plasmaphysik, EURATOM Association, Garching, Germany \\ 3) CompX, Del Mar, CA 92014, USA \\ ${ }^{4)}$ MIT Plasma Science and Fusion Center, Cambridge, Massachusetts 02139, \\ $U S A$
}

(Dated: 25 May 2012)

The paraxial WKB (pWKB) approximation, also called beam tracing method, has been employed in order to study the propagation of the lower hybrid ( $\mathrm{LH})$ waves in a tokamak plasma. This approach reduces the Maxwell's equation to a set of ordinary differential equations, including the ray tracing as a particular case and takes into account also the diffraction effects of the wave. A new code, LHBEAM (Lower Hybrid BEAM tracing), is presented which solves the pWKB equations in a tokamak geometry for arbitrary launching conditions and for analytic and experimental magnetic equilibria. In addition, LHBEAM includes the linear electron Landau damping for the evaluation of the absorbed power density and the reconstruction of the electric field. Some LHBEAM calculations and a comparison with the ray tracing code GENRAY and the full wave solver TORIC-LH are presented.

\section{INTRODUCTION AND BACKGROUND}

Lower hybrid current drive (LHCD) is an efficient tool for non-inductively driving current off-axis in tokamak plasmas. Hence, it may play an important role for the current profile control in the advanced tokamak scenario [REFERENCE!!]. Some unresolved issues in the study of the LH wave propagation still exist, such as the spectral gap problem ${ }^{1}$, i.e., the fact that the parallel (to the magnetic field) refractive index spectrum generated at the plasma edge does not appear to be wide enough to allow the waves to interact with a large number of electrons, and the most recent one related to the "density limit" in the efficiency of $\mathrm{LHCD}^{2,3}$.

The most common approach employed to analyze radio-frequency wave propagation, and specifically $\mathrm{LH}$ wave propagation, is the ray tracing (RT) method based on the WKB approximation or also called the geometrical optics approach ${ }^{4-8}$. This approach is based on the fact that, in most cases of practical interest, the typical inhomogeneity scale of the plasma $L$ is much larger than the radiation wavelenght $\lambda$ and the time $T$ which characterizes the changes in the plasma properties is much larger than the wave period $2 \pi / \omega$. This is referred to as the short-wavelength limit and can be expressed by the introduction of a large dimensionless parameter

$$
\kappa \equiv\left\{\frac{L \omega}{c}, T \omega\right\} \gg 1
$$

( $c$ is the speed of light and $\omega=2 \pi f$ with $f$ the wave frequency). In this situation, the geometrical optics approach is usually employed in order to solve the Maxwell's

\footnotetext{
a) This article is dedicated to the memory of Grigory Pereverzev.

b) nbertell@pppl.gov
}

equations, which are reduced to a set of ordinary differential equations (ODE). The electromagnetic wave beam is then viewed as a bundle of rays, traced independently from each other; along the rays, the wave polarization and amplitude can be calculated. The geometrical optics provides a very powerful tool for solving Maxwell's equations in the short-wavelength limit, since it allows both a simple picture (in terms of rays) of the wave propagation and a direct application to practical problems. In fact, the integration of a set of ordinary differential equations is, from a computational point of view, straightforward. Although the geometrical optics is widely employed in literature, it is important to remark that condition (1) gives a necessary but not a sufficient condition for applying geometrical optics ${ }^{8,9}$. For this reason, there are situations in which the applicability of the geometrical optics is violated, even if the short-wavelength condition is fulfilled: this might be the case of the LH waves which are considered in this work. The necessary and sufficient conditions for the applicability of the geometrical optics have been analyzed in detail by Kravtsov and Orlov ${ }^{8}$. In particular, for the specific case of a homogeneous medium, the sufficient condition (Fresnel condition) for the applicability of the geometrical optics is ${ }^{8}$

$$
\frac{W^{2}}{\ell} \geq \lambda
$$

where $W$ is the wave beam width and $\ell$ is the length of the propagation path. In other words, the condition (2) states that if $W \geq \sqrt{\lambda \ell}$, the diffraction does not play a significant role; on the other hand, if $W \leq \sqrt{\lambda \ell}$, the diffraction must be taken into account. Inequality (2) that is strictly valid for a homogeneous medium, can also be extended qualitatively to the case of inhomogeneous media.

Condition (2) shows that a new scale length comes explicitly into play, namely, the beam width $W$. In particular, for a wave propagation path of length $\ell=\mathcal{O}(L)$, 
it is seen that diffraction effects are significant for

$$
\frac{W}{L}=\mathcal{O}\left(\frac{1}{\sqrt{\kappa}}\right)
$$

This last ordering is the basis of the paraxial WKB (pWKB) approximation also called beam tracing method and is addressed to in this work. In particular, this approach allows one to derive a set of ordinary differential equations as in geometrical optics, taking into account the diffraction effects ${ }^{10-12}$ (see Section II). Techniques other than the pWKB approximation, such as the parabolic wave equation ${ }^{13-15}$ and the quasi-optics approximation $^{16-19}$, which simplify Maxwell's equations to a more tractable set of equations and take into account the diffraction effects, have also been considered in the literature. With respect to the pWKB approximation, these other methods rely on a set of partial differential equations (PDE), the solution of which is, in general, much more difficult, in particular, from a computational point of view. As mentioned above, the propagation of LH wave beams is one case in which the sufficient condition (2) of the geometrical optics, in some situations, is violated $^{20,21}$. In other words, the diffraction effects might play a significant role in the propagation of the LH waves as show in the work of Pereverzev ${ }^{22}$. In addition, the geometrical optics has applicability limitations in the cut-off and caustic regions ${ }^{23}$ which, in the multi-pass regime of LH wave, can play an important role in determining the power absorption profile. At the same time, it is really important to mention not only the asymptotic techniques developed to solve the Maxwell's equations but also the full-wave solvers, which take into account, in principle, all the wave effects. In fact, the kind of limitations of geometrical optics mentioned above motivated the development of the full-wave solvers such as the TORIC-LH ${ }^{24}$ and the LHEAF ${ }^{25}$ codes.

The aim of this paper is to show the application of the pWKB on the LH wave propagation by using a new code, called LHBEAM, which has been developed in order to have an additional tool which has all numerical advantages of the most common ray tracing method and, at the same time, takes into account the effect of the diffraction phenomena. Preliminary results have been shown in Refs. ${ }^{26,27}$. In particular, in LHBEAM, besides the $\mathrm{LH}$ wave beam propagation, the evaluation of the absorbed power density through the linear electron Landau damping and the reconstruction of the electric field are included. This paper is structured as follows. The pWKB approximation is derived in Section II. In Section III, a description of the LHBEAM code is presented. Numerical results are shown in Section IV. Some examples of LH wave beams propagation and power density profiles obtained by LHBEAM with different central electron temperature are shown together with a comparison with the ray tracing code GENRAY ${ }^{28}$ and the full wave solver TORIC-LH for both a circular cross-section equilibrium and an Alcator C-Mod like equilibrium.

\section{PARAXIAL WKB METHOD}

In this section, we write explicitly the pWKB equations for an electromagnetic wave beam, with a Gaussian profile, propagating in a stationary and spatially nondispersive plasma and few comments are given on their physical meaning. The formal derivation of these equations is based on the works of Pereverzev ${ }^{10,11}$ and Poli et al. ${ }^{12}$.

The pWKB method accounts for the detailed form of the wave field since the very beginning, in particular, taking into account the diffractive pattern of the beam cross-section which is characterized by the intermediate scalelength $W$ (see equation (3)). Specifically, the ordering in half-integer powers of $1 / \kappa$ implied by equation (3) is employed to perform an expansion (paraxial expansion) around the beam axis (also called reference ray). In physical terms, the wave field amplitude is supposed to vary across the propagation direction and hence the maximum amplitude is localized around the reference ray and away from it the field is negligible.

The pWKB method provides a solution of Maxwell's equation

$$
\nabla \times \nabla \times \mathbf{E}-\frac{\omega^{2}}{c^{2}} \varepsilon=0
$$

(where $\varepsilon$ is the cold plasma dielectric tensor) in the form

$$
\mathbf{E}=A(\mathbf{r}) \mathbf{e}(\mathbf{r}) e^{i \kappa \bar{s}(\mathbf{r})}=A(\mathbf{r}) \mathbf{e}(\mathbf{r}) e^{i \kappa[s(\mathbf{r})+i \phi(\mathbf{r})]}
$$

where $A(\mathbf{r})$ and e are, respectively, the wave amplitude and the unit polarization vector. Moreover, the functions $\bar{s}(\mathbf{r})=s(\mathbf{r})+i \phi(\mathbf{r})$ is the complex eikonal.

According to the discussion above, the two functions $s(\mathbf{r})$ and $\phi(\mathbf{r})$ are given by an expansion around the reference ray and they read (summation over repeated indices is adopted)

$$
\begin{aligned}
s(\mathbf{r})=s_{0}(\mathbf{r}) & +N_{\alpha}(\tau)\left[x_{\alpha}-x_{\alpha}(\tau)\right]+ \\
& +\frac{1}{2} s_{\alpha \beta}(\tau)\left[x_{\alpha}-x_{\alpha}(\tau)\right]\left[x_{\beta}-x_{\beta}(\tau)\right], \\
\phi(\mathbf{r})= & \frac{1}{2} \phi_{\alpha \beta}(\mathbf{r})\left[x_{\alpha}-x_{\alpha}(\tau)\right]\left[x_{\beta}-x_{\beta}(\tau)\right],
\end{aligned}
$$

where $x_{\alpha}(\tau)$ and $N_{\alpha}(\tau)$ being the coordinates and the components of the refractive index along the reference ray, respectively. $\tau$ is a parameter along the reference ray. In particular, the zero-term in equation (6) is exactly the same as in the geometrical optics approach. On the other hand, in the expansion of $\phi(\mathbf{r})$ the first-order term is equal to zero because, $\phi(\mathbf{r})$, evaluated on the reference ray, is zero by construction and, being, in addition, positive-definite, we also have $\nabla \phi=0$ (for all details the reader is referred to Refs. $\left.{ }^{10-12}\right)$. Then, $x(\tau)$ and $N(\tau)$ satisfy the set of Hamiltonian differential equation of the ray tracing

$$
\frac{d x_{\alpha}}{d \tau}=\frac{\partial H}{\partial N_{\alpha}}, \quad \frac{d N_{\alpha}}{d \tau}=-\frac{\partial H}{\partial x_{\alpha}},
$$


where $H$ is the (real) determinant of the dispersion tensor $\boldsymbol{\Lambda}=\left(\mathbf{N N}-N^{2} \mathbf{I}\right)+\varepsilon^{h}$ with $\mathbf{N}(\equiv c \mathbf{k} / \omega)$ the refractive index, I the identity tensor and $\varepsilon^{h}$ the Hermitian part of the plasma dielectric tensor. The remaining functions $s_{\alpha \beta}$ and $\phi_{\alpha \beta}$ obey the equations

$$
\begin{gathered}
\frac{d s_{\alpha \beta}}{d \tau}=-\frac{\partial^{2} H}{\partial x_{\alpha} \partial x_{\beta}}-\frac{\partial^{2} H}{\partial x_{\beta} \partial k_{\gamma}} s_{\alpha \gamma}-\frac{\partial^{2} H}{\partial x_{\alpha} \partial k_{\gamma}} s_{\beta \gamma}+ \\
-\frac{\partial^{2} H}{\partial k_{\gamma} \partial k_{\delta}} s_{\alpha \gamma} s_{\beta \delta}+\frac{\partial^{2} H}{\partial k_{\gamma} \partial k_{\delta}} \phi_{\alpha \gamma} \phi_{\beta \delta} \\
\frac{d \phi_{\alpha \beta}}{d \tau}=-\left(\frac{\partial^{2} H}{\partial x_{\alpha} \partial k_{\gamma}}+\frac{\partial^{2} H}{\partial k_{\gamma} \partial k_{\delta}} s_{\alpha \delta}\right) \phi_{\beta \gamma}+ \\
-\left(\frac{\partial^{2} H}{\partial x_{\beta} \partial k_{\gamma}}+\frac{\partial^{2} H}{\partial k_{\gamma} \partial k_{\delta}} s_{\beta \delta}\right) \phi_{\alpha \gamma} .
\end{gathered}
$$

In addition, the solution of these equations are subject to the constraints

$$
\frac{\partial H}{\partial x_{\alpha}}+s_{\alpha \beta} \frac{\partial H}{\partial N_{\beta}}=0
$$

and

$$
\phi_{\alpha \beta} \frac{\partial H}{\partial N_{\beta}}=0
$$

so that the number of independent equations, for $s_{\alpha \beta}$ and $\phi_{\alpha \beta}$, is then reduced to six. In particular, equations (11) and (12) can be employed either to reduce the number of equations or as a check of the numerical accuracy.

In order to understand the physical meaning of $s_{\alpha \beta}$ and $\phi_{\alpha \beta}$, which guarantee the description of the wave effects, we consider a simple case in which the beam propagates in free space along the $x$-direction. Then, equations (11) and (12) give the constraints $s_{x x}=s_{x y}=s_{x z}=0$ and $\phi_{x x}=\phi_{x y}=\phi_{x z}=0$, respectively. Moreover, it is supposed that $s_{y z}=\phi_{y z}=0$. In this particular situation, the wave electric field, given by equation (5), becomes (neglecting the amplitude and the polarization)

$$
\begin{aligned}
\mathbf{E} \propto & \exp \left\{i \kappa\left[f(x)+\frac{1}{2}\left(s_{y y}(x) y^{2}+s_{z z}(x) z^{2}\right)\right]+\right. \\
& \left.-\frac{\kappa}{2}\left[\phi_{y y}(x) y^{2}+\phi_{z z} z^{2}\right]\right\}
\end{aligned}
$$

where $f(x)=\int N_{x}(x) d x$ describes the phase evolution on the reference ray (i.e., along the propagation direction) and has the same physical meaning as in geometrical optics. The next step is to introduce the radii of curvature of the wave front $R_{\alpha}$ and the beam widths $W_{\alpha}$, in such a way that the quadratic terms, present in equation (13), can be write in a form which clarifies the physical interpretation of them:

$$
s_{\alpha \alpha}(x) \equiv \frac{\omega / c}{R_{\alpha}(x)}, \quad(\text { no sum over } \alpha),
$$

and

$$
\phi_{\alpha \alpha}(x) \equiv \frac{2}{W_{\alpha}^{2}(x)}, \quad(\text { no sum over } \alpha),
$$

along the $y$ and $z$-direction. Therefore, the symmetric tensor $s_{\alpha \beta}$ is connected with the curvature of the wave front, whereas $\phi_{\alpha \beta}$ describes the beam profile. In addition, from equation (13), one can note that the contour levels of the amplitude profile are given by a quadratic form whose axes are aligned with the $y$ and $z$ axes of the laboratory system. Such a quadratic form $\phi_{y y} y^{2}+\phi_{z z} z^{2}$ is positive definite and its contour levels are ellipses (they can become circles when two beam widths are the same along the two directions). The ellipse having semi-axes equal to $W_{\alpha}$ will be called attenuation ellipse. An analogous analysis can be carried out for phase fronts that, in particular, are characterized by the quadratic form $s_{y y} y^{2}+s_{z z} z^{2}$. When $s_{y z} \neq 0\left(\phi_{y z} \neq 0\right)$, one has the rotation of the principal radii of curvature (the principal widths) with respect to the fixed reference frame. For instance, in the case of inhomogeneous media, during the propagation of the wave beam, the evolution of the attenuation ellipse can be quite complicated, in fact the attenuation ellipse can rotate and change the shape. In Section IV A, a 3D plot of a LH beam is shown in which the evolution of this attenuation ellipse can be clearly seen.

Finally, in the framework of the pWKB method, one can get, analogously to the geometrical optics, the wave energy transport equation

$$
\nabla \cdot\left(\mathbf{v}_{g} U\right)=-2 \gamma U
$$

where $\mathbf{v}_{g}\left(\propto \mathbf{V}\left(\equiv \frac{\partial H}{\partial \mathbf{N}}\right)\right)$ is the group velocity, $U \propto|A|^{2}$ is the energy density and $\gamma=\mathbf{e}^{*} \cdot \varepsilon^{a} \cdot \mathbf{e}$ the absorption coefficient ( $\varepsilon^{a}$ is the anti-Hermitian part of the dielectric tensor). In addition, the polarization vector $\mathbf{e}$ is obtained, as in geometrical optics, by the equation $\boldsymbol{\Lambda} \cdot \mathbf{e}=0$. It is important to stress that, although the form of the wave energy transport equation (16) is the same as in the geometrical optics, the physical meaning is different due to that the pWKB approach describes the evolution of the energy density of the whole beam and not only for a single ray as in the geometrical optics. This means that, equation (16) takes into account, in a intuitive point of view, not only the contribution of the energy density flux along the geometrical optics rays, but also the transverse contribution with respect to them, which is neglected in the standard geometrical optics. More specifically, the effects of diffraction enters, e.g., in equation (16) through the term

$$
\nabla \cdot \mathbf{V}=\frac{\partial}{\partial x_{\alpha}} \frac{\partial H}{\partial N_{\alpha}}+\frac{\partial^{2} s}{\partial x_{\alpha} \partial x_{\beta}} \frac{\partial^{2} H}{\partial N_{\alpha} \partial N_{\beta}},
$$

in which the dependence on the matrix $s_{\alpha \beta}=$ $\partial^{2} s / \partial x^{\alpha} \partial x^{\beta}$ is evident, this quantity being coupled to the beam widths as discussed above. For the specific case of an isotropic medium, the analysis of such a contribution has been carried out in Ref. ${ }^{29}$. 


\section{CODE DESCRIPTION}

LHBEAM is a Fortan 90 code part of which is based on the TORBEAM code which is the first code that applied the pWKB approximation to Gaussian wave beams with frequencies in the electron-cyclotron frequency range in a fusion plasma ${ }^{30}$. The aim of LHBEAM is to solve numerically the 18 ordinary differential equations of the pWKB approximation, in particular, 6 equations for the reference ray (cf. equations (8)), $6+6$ equations for the components of the symmetric matrices $s_{\alpha \beta}, \phi_{\alpha \beta}$ (cf. equations (9), (10)) and $1+1$ equations for the wave amplitude and the power. In addition, the dispersion equation $H=0$, which in the pWKB approximation holds on the reference ray only, and the six constraints (cf. equations (11),(12)) are used to prescribe consistent initial conditions for the beam parameters and, during the run, as a check of solution accuracy. With reference to equations (8)-(10), in the LHBEAM code the dispersion function $H$ can be chosen to be either the full electromagnetic dispersion function

$$
\begin{aligned}
H_{E L M}= & S N_{\perp}^{4}-\left[\left(S-N_{\|}^{2}\right)(P+S)-D^{2}\right] N_{\perp}^{2}+ \\
& +P\left[\left(S-N_{\|}^{2}\right)^{2}-D^{2}\right]
\end{aligned}
$$

or the electrostatic dispersion function

$$
H_{E L S}=S N_{\perp}^{2}+P N_{\|}^{2}
$$

where $N_{\perp}\left(N_{\|}\right)$is the perpendicular (parallel) component of the refractive index with respect to magnetic field and the plasma dielectric tensor is computed in the cold plasma limit and in the range of LH frequency approximation (i.e., $\omega_{c i}^{2} \ll \omega^{2} \ll \omega_{c e}^{2}$ ). In particular, the elements of the cold dielectric tensor are ${ }^{31}$

$$
S=1+\frac{\omega_{p e}^{2}}{\omega_{c e}^{2}}-\frac{\omega_{p i}^{2}}{\omega^{2}}, D=\frac{\omega_{p e}^{2}}{\omega \omega_{c e}}, P=1-\frac{\omega_{p e}^{2}}{\omega^{2}}-\frac{\omega_{p i}^{2}}{\omega^{2}},
$$

where

$$
\omega_{\mathrm{pe}}^{2}(\mathbf{r}) \equiv \frac{4 \pi n_{e}(\mathbf{r}) e^{2}}{m_{\mathrm{e}}}, \quad \omega_{\mathrm{ce}}(\mathbf{r}) \equiv \frac{e B(\mathbf{r})}{m_{\mathrm{e}} c}
$$

and

$$
\omega_{\mathrm{pi}}^{2}(\mathbf{r}) \equiv \sum_{\mathrm{i}=\text { species }} \frac{4 \pi n_{i}(\mathbf{r}) Z_{\mathrm{i}} e^{2}}{m_{\mathrm{i}}} \approx \frac{4 \pi n_{e}(\mathbf{r}) Z_{\mathrm{eff}} e^{2}}{m_{\text {main }}}
$$

are the square of the electron plasma frequency, the electron cyclotron frequency and the square of the ion plasma frequency, respectively $\left(n_{\mathrm{e}}\left(n_{\mathrm{i}}\right)\right.$ is the electron (ion) density, $e$ the absolute value of the electron charge and $m_{\mathrm{e}}$ $\left(m_{\mathrm{i}}\right)$ the electron (ion) mass, $\mathbf{B}$ the confinement magnetic field, $Z_{\text {eff }}$ the effective charge of the plasma and $m_{\text {main }}$ the mass of the main ion species). In the case of full electromagnetic dispersion function, equation (18) is just the determinant of the dispersion tensor, i.e., no mode selection is operated at this level. With this choice, the derivatives of $H$ can be calculated in a simpler way. The slow wave mode (corresponding to the LH waves) is selected imposing initial conditions that satisfy the dispersion equation $H=0$ and controlling that this dispersion equation is then fulfilled along the whole propagation path.

Regarding the evaluation of the power absorption of LH waves, in LHBEAM a Maxwellian plasma is considered therefore the main contribution of the imaginary part of the dispersion relation due to electrons corresponds to the linear electron Landau damping of the LH waves. More specifically, the absorbed power is calculated according to the equation

$$
\frac{d P}{d \tau}=-2 \gamma_{\mathrm{ELD}} P
$$

where $\gamma_{\text {ELD }}$ corresponds to the linear electron Landau damping given by ${ }^{32-34}$

$$
\begin{aligned}
\gamma_{\mathrm{ELD}}= & 2 \sqrt{\pi}\left(\frac{\omega_{\mathrm{pe}}}{\omega}\right)^{2} \frac{\omega}{c} \frac{c^{3}}{N_{\|}^{3} v_{\mathrm{th}}^{3}} \times \\
& \times\left[\left(S-N_{\|}^{2}\right)\left(S-N^{2}\right)-D^{2}\right] \times \\
& \times\left|\frac{\partial H}{\partial \mathbf{N}}\right|^{-1} \exp \left[-\frac{c^{2}}{v_{\mathrm{th}}^{2} N_{\|}^{2}}\right] .
\end{aligned}
$$

In equation $(24), v_{\text {th }} \equiv \sqrt{2 T_{\mathrm{e}} / m_{\mathrm{e}}}$ is the electron thermal velocity, respectively.

The plasma equilibrium is prescribed both analytically and from experimental data. For the former case, an analytical representation of the poloidal and toroidal components of the magnetic field is implemented taking into account the Shafranov shift and the elongation of the plasma (see Ref. ${ }^{30}$ ). The radial profile of the density and the temperature are given in the following form

$$
f(r)=\left(f_{0}-f_{\text {edg }}\right)\left[1-\left(\frac{r}{a}\right)^{e 1}\right]^{e 2}+f_{\text {edg }}
$$

where $f(r)$ indicates both the density $(n(r))$ and the temperature $(T(r))$ and the subscripts " 0 " and "edg" denotes the values at the plasma core and plasma edge, respectively. In equation (25), $a$ is the minor radius of the plasma torus and the $f_{0}, f_{\text {edg }}, e 1$ and $e 2$ are given as input parameters for both density and temperature. For the experimental case, the magnetic configuration is provided numerically by assigning the (Cartesian) components of the static magnetic field $\mathbf{B}$ and a flux coordinate $\psi$ on a grid in the poloidal plane $(x, z)$. Density and temperature are also prescribed numerically. They are given as functions of $\psi$.

\section{RESULTS AND BENCHMARK}

In this section we show some results obtained from LHBEAM and a benchmark with the ray tracing code 
(a)

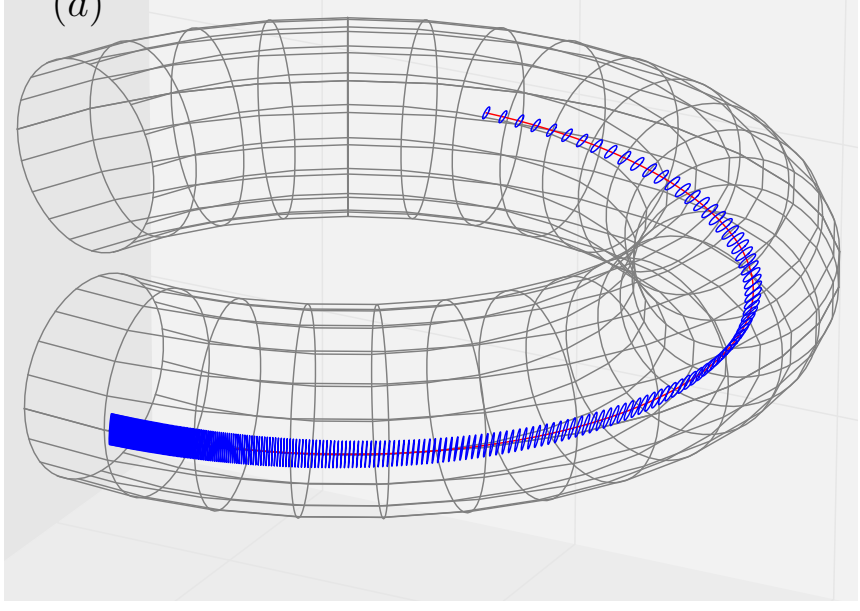

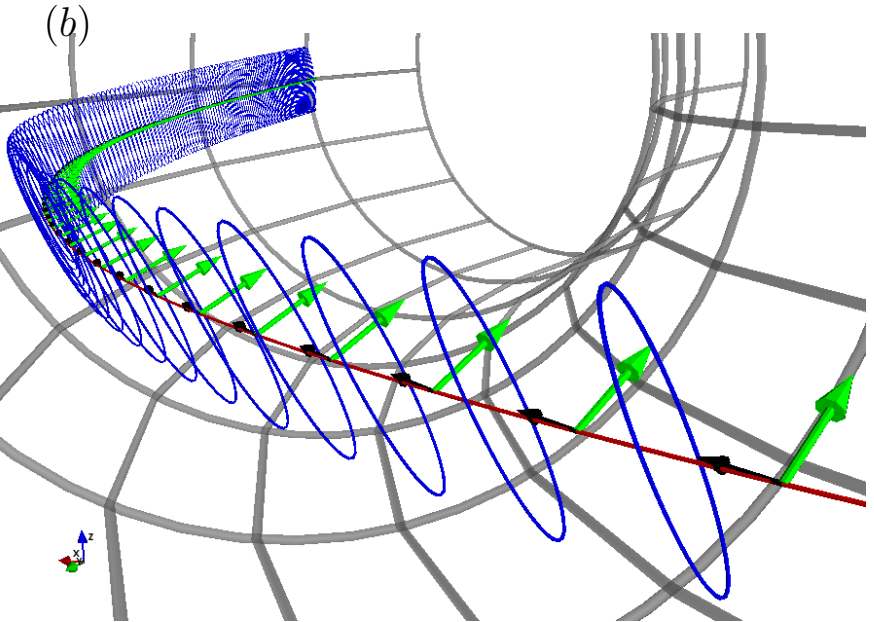

FIG. 1. (a) 3D evolution of the LH wave beam for $T_{\mathrm{e}, 0}=5 \mathrm{keV}$ and $N_{\|, 0}=2.5$. Attenuation ellipses (mentioned in Section II) are plotted in blue and the beam axis in red. (b) A zoom-in of figure (a) in another point of view. The green arrow represents the wave vector direction along the LH propagation pointing to the high field side and the black arrow represents the direction of the magnetic field opposite to the direction of the LH wave beam.

GENRAY ${ }^{28}$ and the full wave solver TORIC-LH ${ }^{24}$ regarding the beam axis trajectory, the evolution of the refractive index along the propagation and the power density profile.

\section{A. Numerical results}

The tokamak plasma equilibrium adopted in this subsection has a circular cross-section with a major radius $R_{0}=64 \mathrm{~cm}$ and a minor radius $a=16.5 \mathrm{~cm}$. The magnetic field $B\left(R_{0}\right)=8 \mathrm{~T}$ and the plasma current $I_{\mathrm{p}}=400$ $\mathrm{kA}$. Temperature and density profiles have parabolic profile following equation (25). In particular, the central and the edge electron density are $n_{\mathrm{e}, 0}=5 \times 10^{19} \mathrm{~m}^{-3}$ and $n_{\mathrm{e}, \mathrm{edg}}=1 \times 10^{19} \mathrm{~m}^{-3}$. With reference to the temperature, we consider three different values for the central electron temperature such as $T_{\mathrm{e}, 0}=3,5,10 \mathrm{keV}$ whereas the edge electron temperature, for all three cases, is $T_{\text {e,edg }}=0.5 \mathrm{keV}$. The initial wave front of the LH beam is considered flat and the beam width is $W=3 \mathrm{~cm}$ corresponding to a $6 \mathrm{~cm}$ height antenna located on the low field side of the torus. Finally, the frequency of LH beam is $4.6 \mathrm{GHz}$ and the initial value of parallel refractive index is $N_{\|, 0}=2.5$. The input power is assumed to be 1 MW and the full electromagnetic dispersion function (cf. equation (18)) is adopted.

Figure 1 shows a 3D propagation of the $\mathrm{LH}$ wave beam for $T_{\mathrm{e}, 0}=5 \mathrm{keV}$ launched in equatorial plane. In Figure 1 the evolution of the attenuation ellipse mentioned in Section II is plotted in blue line showing its rotation during the LH beam propagation whereas the beam axis trajectory is plotted in red line. A zoom-in of Figure 1(a) from another point of view with respect to Figure 1(a) is shown in Figure 1(b). Here, one case see not only the evolution of the attenuation ellipse but also the direction of the wave vector along the beam axis represented by the green arrows pointing to the high field side and the direction of the magnetic field represented by the black arrows pointing basically opposite to the LH wave beam. In this figure it appear clearly one of the main feature of the LH wave, namely the fact that LH wave tends to propagate parallel to the magnetic field (although, in this specific case, in the opposite direction) and the wave vector tends to be perpendicular to the static magnetic field. It is less clear to see in the figure that the LH wave is backward wave (i.e. the phase and group velocities across the magnetic field are oppositely directed) although this is the case, in fact, there is a small projection of the wave vector in the opposite direction of the beam axis which corresponds to the direction of the group velocity. In order to illustrate even better the LH wave beam, the most common polidal and toroidal wave beam propagation, typically shown in literature, are shown in Figure 2 (a) and 2(b), respectively. The light blue lines represent the projections of the attenuation ellipses on the poloidal and toroidal section, respectively and the red line is the beam axis as shown in Figure 1.

Another feature of LHBEAM is the possibility to reconstruct the wave electric field. Since LHBEAM is written in Cartesian coordinates, it provides the Cartesian coordinates of the electric field. In order to represent the wave electric field in a similar way of the full-wave solvers which typically consider only a single wave toroidal mode number $n_{\varphi}$, we have performed a Fourier transform of the electric field (which in the pWKB framework takes naturally into account also the toroidal width of the antenna) in the toroidal component $\varphi$ and we have kept the dominant component in $n_{\varphi}$-spectrum. A numerical result of this procedure is shown in Figure 3 where it 

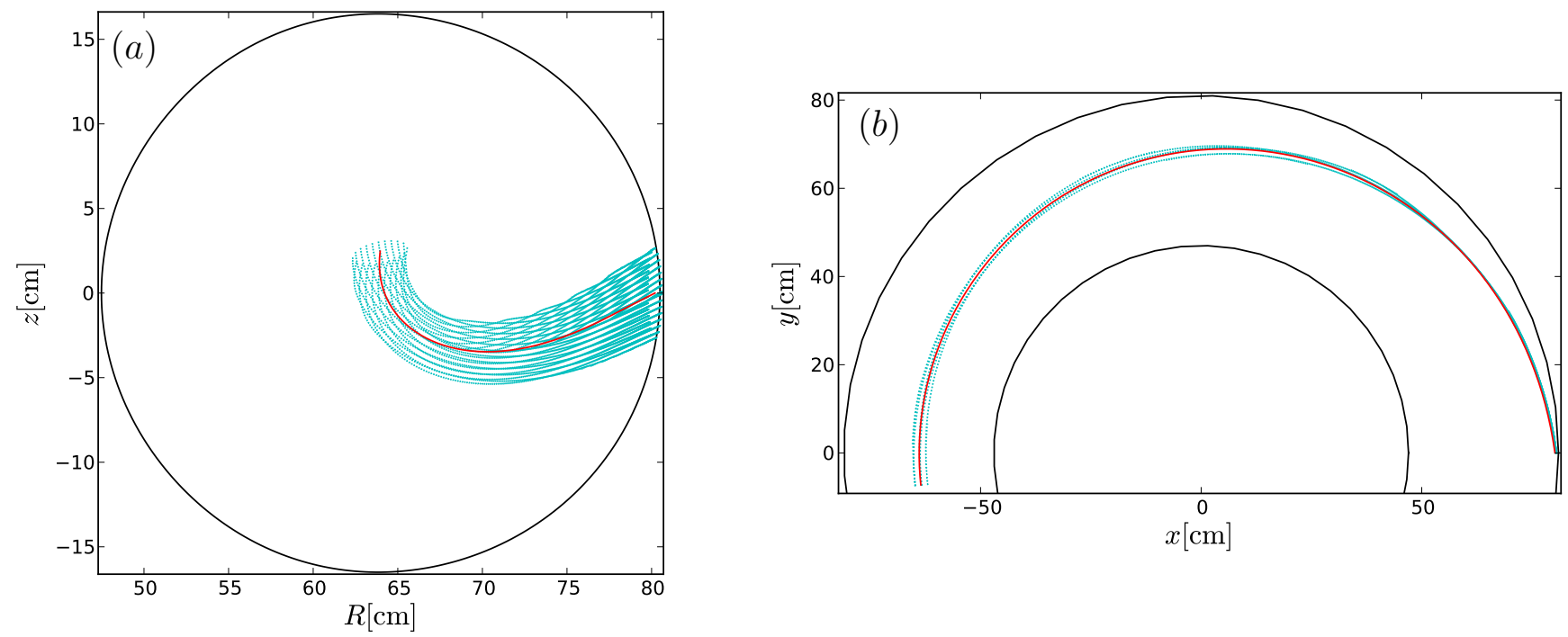

FIG. 2. (a) Poloidal wave beam propagation and (b) toroidal wave beam propagation corresponding to Figure 1 . The red curve represents the beam axis and the light blue curves represent the projections of the attenuation ellipses on the poloidal and toroidal section, respectively.

appears that the magnitude of the parallel (with respect to the magnetic field) component of the complex electric field for $T_{e, 0}=3 \mathrm{keV}$ (figure $3(\mathrm{a})$ ), $T_{e, 0}=5 \mathrm{keV}$ (figure $3(\mathrm{~b})$ ) and $T_{e, 0}=10 \mathrm{keV}$ (figure $3(\mathrm{c})$ ). The dominant component of the $n_{\varphi}$-spectrum, for these cases, it is found to be $n_{\varphi}=-191$. This number is slightly different in comparison with the input parameter $n_{\varphi}=-196$ for the corresponding case running TORIC-LH. This is due to (i) the lack of any detailed antenna description in the current version of LHBEAM with the consequence that we cannot have the exact initial conditions with respect to TORIC-LH; (ii) the numerical tolerances of the numerical Fourier transform of the field. In fact, it is worth noting that the $n_{\varphi}$-spectrum obtained from LHBEAM is well peaked around the dominant component and such difference on the dominant component in $n_{\varphi}$ is basically negligible. From Figure 3, one can see, as expected, that the parallel component of the electric field follows the beam trajectory and its maximum lies on the beam axis and decreases away from it.

\section{B. Benchmark with GENRAY and TORIC-LH}

In order to validate the pWKB code for LH wave, LHBEAM, we need to compare this code with other independent codes. To compare carefully different codes is not an easy task, in particular, when each code is based on a different physical model and different kind of approximations. Here, a code validation is presented regarding the trajectory of the propagation and the evolution of the parallel and perpendicular components of the refractive index with the ray tracing code GENRAY ${ }^{28}$ and absorbed power with both GENRAY and the full-wave solver TORIC- $\mathrm{LH}^{24}$. As mentioned in the previous section, the ray tracing approximation is a particular case of the pWKB method. In fact, the beam axis obeys to the ray tracing equations (cf. equations (8)). Therefore, we can compare directly the trajectory of the beam axis calculated by LHBEAM with the trajectory of a single ray calculated by the ray tracing code GENRAY together with the evolution of the refractive index along the beam axis. A preliminary code validation has been done in the work of Bertelli et al. $2008^{27}$ by using an analytical equilibrium with the ray tracing code $\mathrm{C}^{3} \mathrm{PO}^{35,36}$, here we show two cases by using both a circular crosssection equilibrium used in the previous subsection and an Alcator C-Mod like equilibrium. Figure 4 shows the poloidal (Figure 4(a)) and toroidal (Figure 4(b)) projections of the beam axis obtained by LHBEAM in full red curve together with the trajectory of a single ray calculated by GENRAY in dashed blue curve assuming $N_{\|}=2.5$ and parabolic plasma profiles with $T_{\mathrm{e}}=3 \mathrm{keV}$. It appears clearly a very good agreement between these two independent codes, in fact, it is hard to distinguish the two curves plotted. The same behavior appears also in Figure 5 where the parallel (Figure $5(\mathrm{a})$ ) and perpendicular (Figure 5(b)) components (with respect to the magnetic field) of the refractive index as a function of the major radius, $R$, are plotted, respectively. With reference to the Alcator C-Mod like equilibrium, the same really good agreement mentioned above appears in Figures 6 and 7 where again the beam axis trajectory and the evolution of the refractive index (parallel and per- 

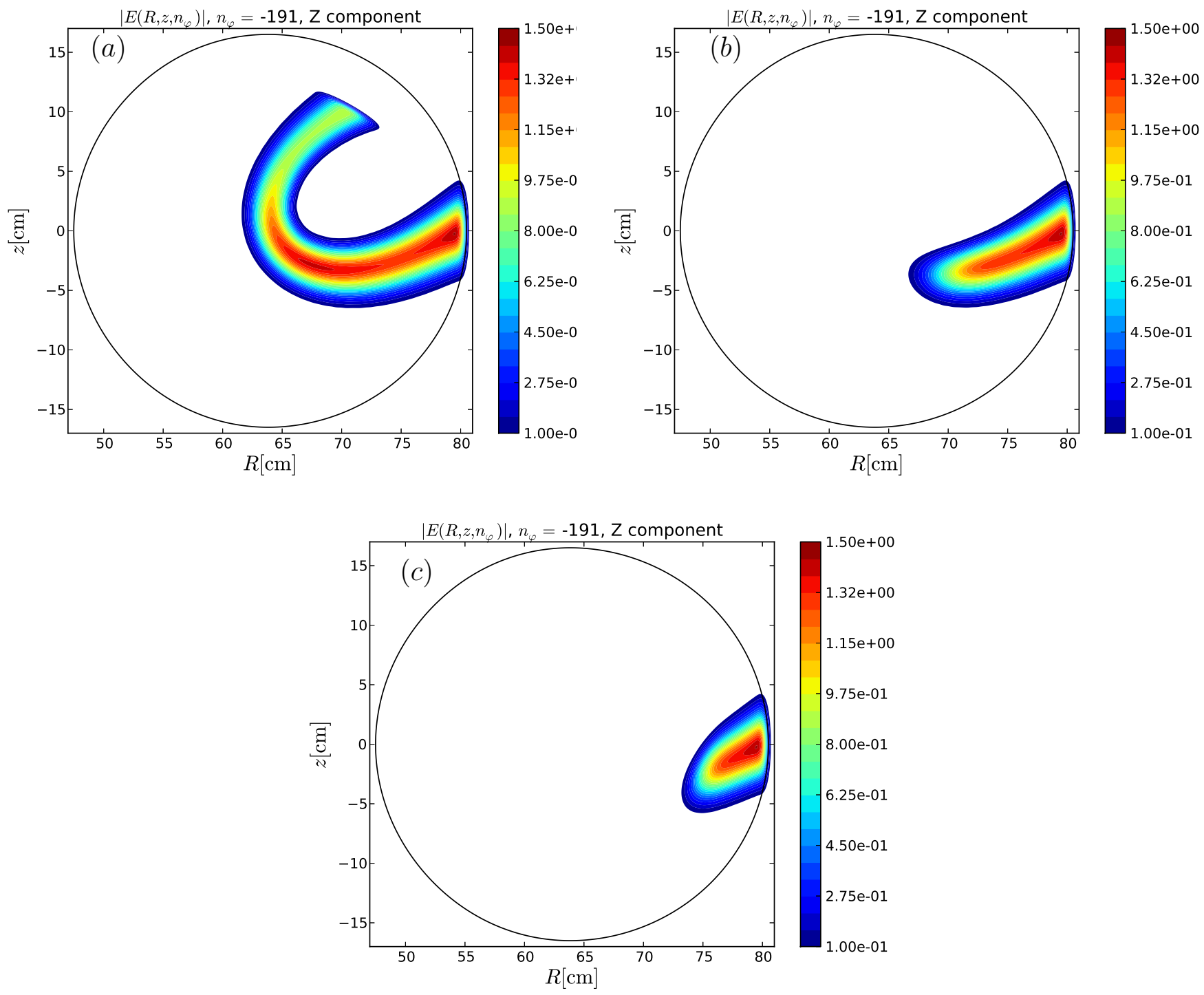

FIG. 3. Magnitude of the parallel component of the complex electric field for three different cases: $T_{\mathrm{e}, 0}=3 \mathrm{keV}$ (figure (a)), 5 $\mathrm{keV}$ (figure (b)), $10 \mathrm{keV}$ (figure (c)). $n_{\varphi}=-191$ is the toroidal wave number and its value corresponds to dominant component of the spectrum.

pendicular components) are plotted. Therefore LHBEAM and GENRAY have really similar results in terms of the trajectory and the evolution of the refractive index during the beam propagation although they have two different interpolation method of the experimental equilibrium data. Finally, in order to increase further our confidence in the LHBEAM calculations an additional comparison regarding the power density profile is shown in Figure 8 with, besides GENRAY, a full wave code TORIC-LH. Specifically, Figure 8 shows the power density as a function of the square root of the normalized poloidal flux, $\rho_{\psi}$. Three cases are shown for different central electron temperature, namely, $T_{\mathrm{e}, 0}=3 \mathrm{keV}$ (red curves), $T_{\mathrm{e}, 0}=5 \mathrm{keV}$ (green curves) and $T_{\mathrm{e}, 0}=10 \mathrm{keV}$ (black curves) by using circular cross-section equilibrium and parabolic profiles described above. Solid, dashed and dashed-dotted lines correspond to LHBEAM, TORIC-LH and GENRAY results, respectively. From Figure 8, it appears a good agreement among codes. In particular, for $T_{\mathrm{e}, 0}=5,10 \mathrm{keV}$, the results are very similar. It is worth noting that the $T_{\mathrm{e}, 0}=3$ $\mathrm{keV}$ case would require to take into account a reflection at the wall because the power is not fully absorbed in a single pass therefore $T_{\mathrm{e}}=3 \mathrm{keV}$ case corresponds to a limit case for LHBEAM. Despite the fact that reflections are not yet treated in LHBEAM, the agreement between the pWKB approximation and the full-wave approach is quite good. Slightly differences appear between LHBEAM and TORIC-LH, in particular, in the range $\rho_{\psi} \gtrsim 1.8$ be- 

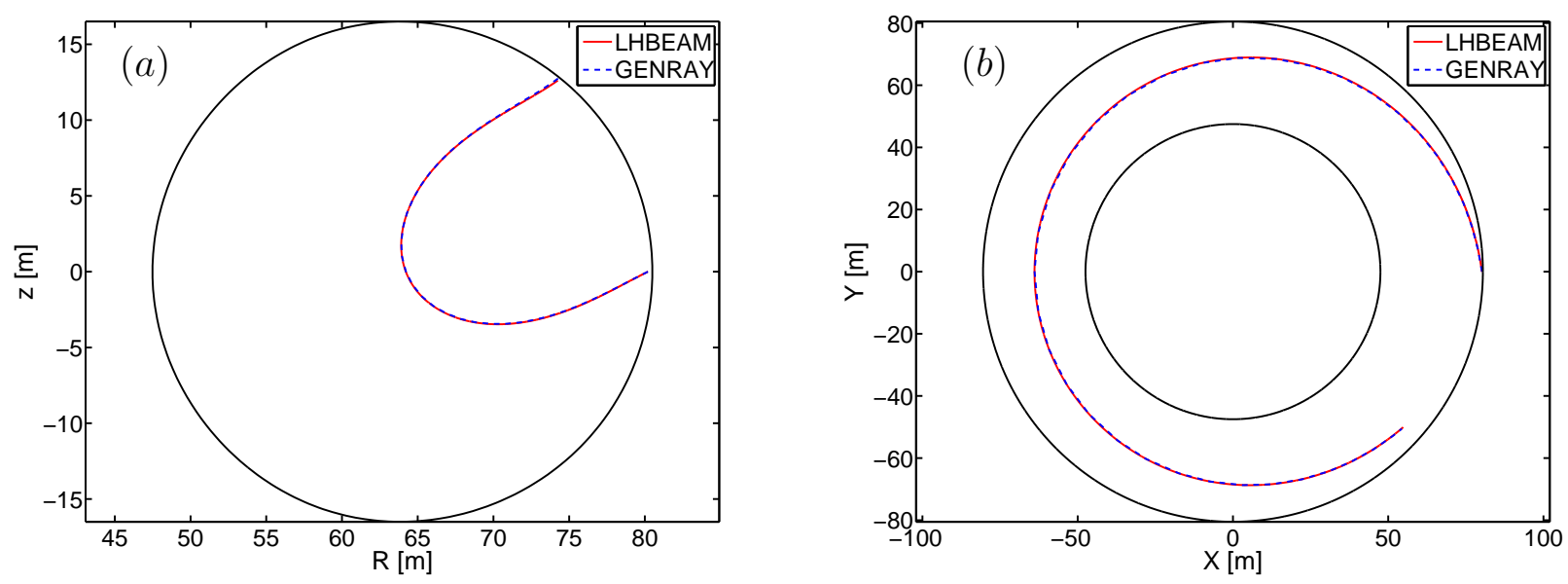

FIG. 4. Poloidal (a) and toroidal (b) projection of the trajectory of the LH beam axis calculated by LHBEAM (full red curve) and the trajectory of a single ray calculated by GENRAY ${ }^{28}$ (dashed blue curve). Circular cross-section equilibrium, parabolic plasma profiles and the initial value of $N_{\|}=2.5$ and electron temperature $T_{\mathrm{e}}=3 \mathrm{keV}$ are assumed.
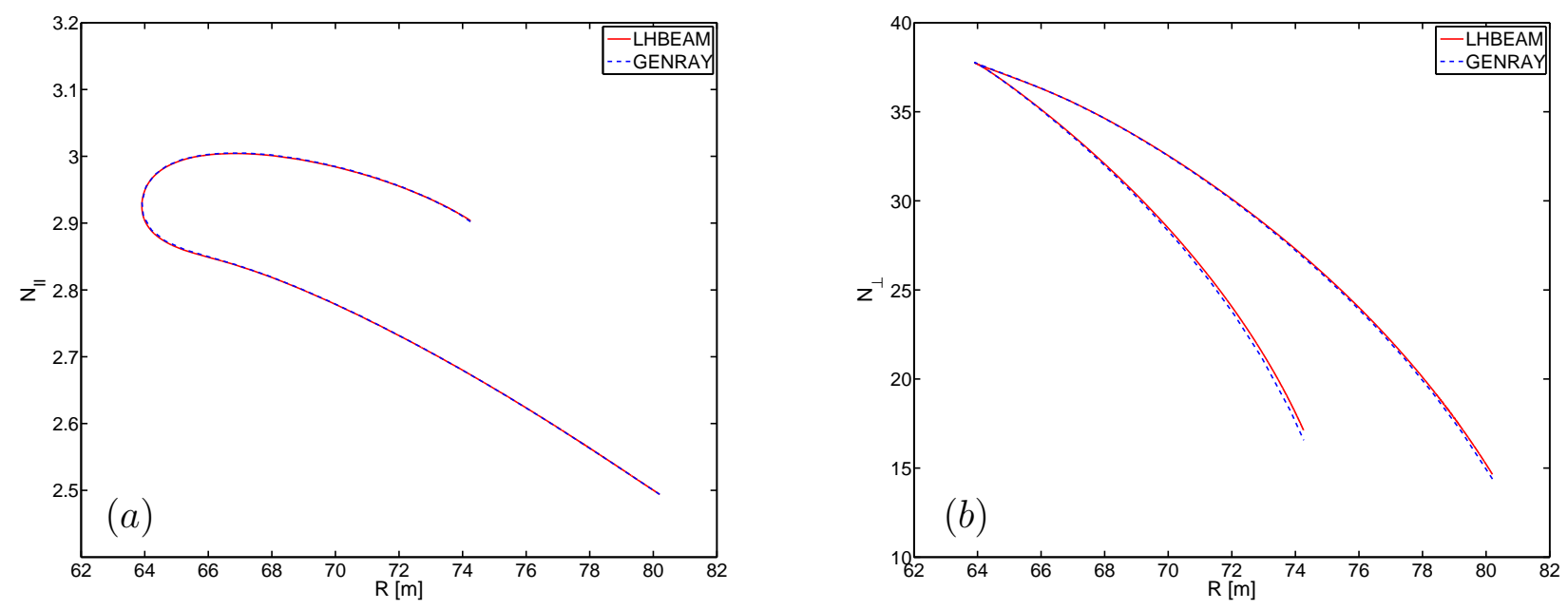

FIG. 5. Parallel (a) and perpendicular component of the refractive intex as a function of $R$, corresponding to the case of Figure 4, calculated by LHBEAM (full red curve) and GENRAY ${ }^{28}$ (dashed blue curve).

cause of the missing small amount absorbed power after the reflection of the LH wave beam at the wall. The differences with GENRAY might occur due to the lack of the ray tracing model to describe in details some wave effects such as diffraction effect. However, for the strong absorption regime, the location of the peak of the power density and its profiles are in very good agreement among codes. In particular, the fact that for $T_{\mathrm{e}, 0}=5,10 \mathrm{keV}$ the agreement between the pWKB technique and the ray tracing approximation is quite good, it might mean that, for the strong absorption regime, the diffraction effect in the real space (in other words, the spatial broadening) for the absorbed power might not play a significant role. On the other hand, the consequences of the spectral broadening in the absorption has to be still investigated both in the weak and strong absorption and it can help to solve a still open issue such as, the so called, "spectral gap problem". A separate work on this topic will be addressed.

\section{SUMMARY AND DISCUSSION}

In this paper the numerical application of the pWKB method to the LH wave propagation has been addressed. In particular, the main pWKB equations have been presented together with their physical interpretation and a description of LHBEAM, a numerical code based on this model for LH beam wave, has been provided. A 3D reconstruction of the LH wave Gaussian beam has been done together with a reconstruction of the electric field. Unlike the electric field obtained from a full-wave solver 


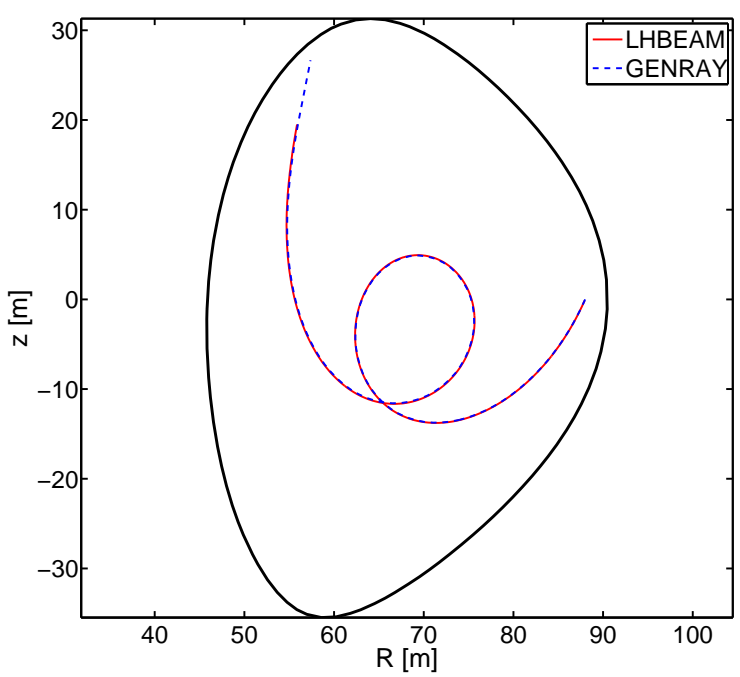

FIG. 6. Poloidal projection of the trajectory of the LH beam axis calculated by LHBEAM (full red curve) and the trajectory of a single ray calculated by GENRAY ${ }^{28}$ (dashed blue curve) . Alcator C-Mod like equilibrium and the initial value of $N_{\|}=5$ are assumed.

which typically makes use of a single wave toroidal number, the electric field based on the pWKB approximation takes into account, naturally, the whole $n_{\varphi}$-spectrum, in other words, the finite size of the antenna, not only in the poloidal but also in the toroidal direction. However, in this work has been shown only the dominant component of the of the $n_{\varphi}$-spectrum for the electric field, in fact, the $n_{\varphi}$-spectrum is found to be really peaked around this dominant component.

A detailed validation of LHBEAM has been also presented. In particular, a comparison with the ray tracing code GENRAY with reference to the trajectory of the beam axis and the evolution of the refractive index has been analyzed for both a circular-cross section with parabolic plasma profiles and an Alcator C-Mod like equilibrium and plasma profiles. A really good agreement in both cases is found. In addition, a comparison of the power density profile with GENRAY and the full-wave solver TORIC-LH has been studied showing again a good agreement among codes. In this work it has been considered only the strong absorption regime (also called the single pass absorption regime) in order to avoid the cut-offs and reflections which need a specific analysis and treatment in the pWKB framework. For the very strong absorption cases $\left(T_{\mathrm{e}, 0}=5,10 \mathrm{keV}\right.$ analyzed in the previous section), the results obtained from pWKB approximation, ray-tracing and full-wave approach are very similar suggesting that the spatial broadening of the beam due to the diffraction effect might be not so significant for the calculations of the absorbed power. In a limit case for the strong absorption regime $\left(T_{\mathrm{e}, 0}=3 \mathrm{keV}\right)$, the $\mathrm{pWKB}$ seems to be more accurate than the ray-tracing method although the lack of reflection treatment in the current version of LHBEAM. This is, in fact, a promising result.

Still it remains to investigate the effect of spectral broadening in the absorbed power due to the diffraction effect both in the weak and strong absorption together with a specific treatment of the caustics and cutoffs which are expected to be important in order to have an accurate model, in particular, in the weak absorption regime. These topics are subject of a future work. One the other side, the agreement between LHBEAM and TORIC-LH presented in this work it seems to go in the direction that the pWKB technique can capture part of the physics described in the full-wave solver. It is important, in fact, to mention that the application of the pWKB technique requires much less computation time and resources with respect to the full-wave approach and it could be really convenient to have an accurate reduced model that executes much faster the LH propagation and absorption calculations.

\section{ACKNOWLEDGMENTS}

This research is supported by the U.S. Department of Energy under contract number ...

${ }^{1}$ P. T. Bonoli and R. C. Englade, Phys. Fluids 29, 2937 (1986).

${ }^{2}$ G. Wallace and et al, Phys. Plasmas 17, 082508 (2010).

${ }^{3}$ R. Cesario and et al, Plasma Phys. Control. Fusion 53, 085011 (2011).

${ }^{4}$ S. Weinberg, Phys. Rew. 126, 1899 (1962).

${ }^{5}$ L. D. Landau and E. M. Lifshitz, The Classical Theory of Fields (Pergamon Press, Oxford, 1987).

${ }^{6}$ I. B. Bernstein, Phys. Fluids 18, 320 (1975).

${ }^{7}$ M. Brambilla and A. Cardinali, Plasma Physics 24, 1187 (1982).

${ }^{8}$ Y. A. Kravtsov and Y. I. Orlov, Geometrical Optics of Inhomogeneous Media, Vol. 6 (Springer Series on Wave Phenomena, 1990).

${ }^{9}$ Y. A. Kravtsov and Y. I. Orlov, Sov. Phys. Usp. 23, 750 (1980).

${ }^{10}$ G. V. Pereverzev, Reviews of Plasma Physics, edited by B. B. Kadomtsev, Vol. 19 (1996).

${ }^{11}$ G. V. Pereverzev, Phys. Plasmas 5, 3529 (1998).

${ }^{12}$ E. Poli, G. V. Pereverzev, and A. G. Peeters, Phys. Plasmas 6, 5 (1999).

${ }^{13}$ V. A. Fock, Electromagnetic Diffraction and Propagation Problems (Pergamon Press, New York, 1965).

${ }^{14}$ G. V. Permitin and A. I. Smirnov, JETP 82, 395 (1996).

${ }^{15}$ A. I. Smirnov and E. Y. Petrov, in Proceedings of the 26th EPS Conference on Plasma Physics and Controlled Fusion, Vol. 23J (1999) p. 1797.

${ }^{16}$ S. Choudhary and L. B. Felsen, Proc. IEEE Trans. Antennas Propag. AP-21, 827 (1973).

${ }^{17}$ E. Mazzucato, Phys. Fluids B 1, 1855 (1989).

${ }^{18}$ S. Nowak and A. Orefice, Phys. Fluids B 5, 1945 (1993).

${ }^{19}$ S. Nowak and A. Orefice, Phys. Plasmas 1, 1242 (1994).

${ }^{20}$ G. V. Pereverzev, in 20th EPS Conference on Contr. Fusion and Plasma Phys., Vol. 17C, Part III (1993) p. 885.

${ }^{21}$ A. Cardinali and et al, Phys. Plasmas 14, 112506 (2007).

${ }^{22}$ G. V. Pereverzev, Nucl. Fusion 32, 1091 (1992).

${ }^{23}$ A. S. Richardson, P. T. Bonoli, and J. C. Wright, Phys. Plasmas 17, 052107 (2010).

${ }^{24}$ J. C. Wright and et al, Phys. Plasmas 16, 072502 (2009).

${ }^{25}$ S. Shiraiwa, Phys. Plasmas 17, 056119 (2010).

${ }^{26} \mathrm{~N}$. Bertelli and et al, in 34th EPS Conf. on Plasma Phys., Vol. 31F (2007) p. P5.051.

${ }^{27}$ N. Bertelli and et al, in AIP Conference Proceedings, Vol. 1069 (2008) pp. 259-264.

28 A. P. Smirnov and R. W. Harvey, in Bull. Am. Phys. Soc., Vol. 40 (1995) p. 1837. 

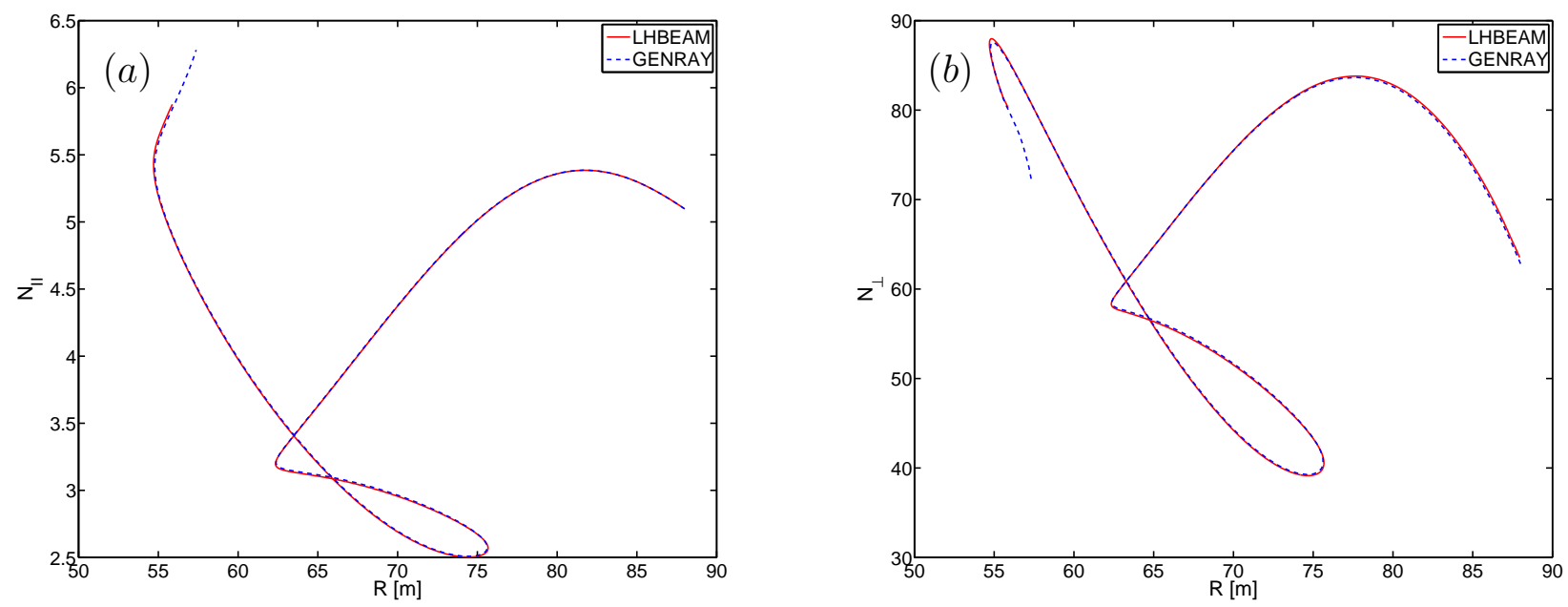

FIG. 7. Parallel (a) and perpendicular (b) component of the refractive index as a function of $R$, corresponding to the case of Figure 6, calculated by LHBEAM (full red curve) and GENRAY ${ }^{28}$ (dashed blue curve).

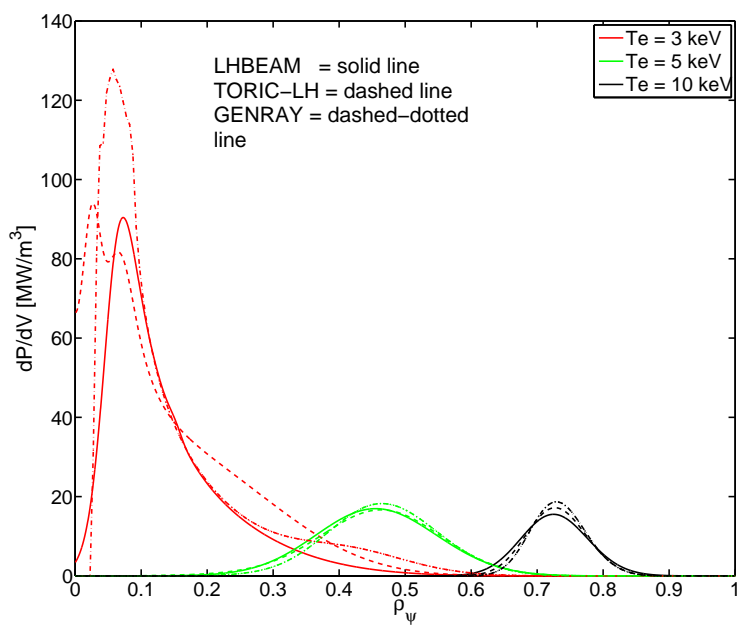

FIG. 8. Power absorption profile as a function of the square root of the normalized poloidal flux, $\rho_{\psi}$ as calcluated by LHBEAM (solide lines), GENRAY (dashed-dotted lines) and TORIC-LH (dashed lines) for $T_{\mathrm{e}, 0}=3 \mathrm{keV}$ (red curves), $5 \mathrm{keV}$ (green curves) and $10 \mathrm{keV}$ (black curves). Input power is 1 MW.
${ }^{29}$ M. Bornatici and O. Maj, Plasma Phys. Control. Fusion 45, 707 (2003).

${ }^{30}$ E. Poli, A. G. Peeters, and G. V. Pereverzev, Comp. Phys. Comm. 136, 90 (2001).

${ }^{31}$ T. H. Stix, Waves in Plasmas (American Institute of Physics, NY, 1992).

${ }^{32}$ P. T. Bonoli, IEEE Trans. on Plasma Science PS-12, 95 (1984).

${ }^{33}$ M. Spada and et al, Nucl. Fusion 31, 447 (1991).

${ }^{34}$ F. Imbeaux and Y. Peysson, Plasma Phys. Control. Fusion 47, 2041 (2005).

${ }^{35}$ Y. Peysson, J. Decker, and V. Basiuk, in 34th EPS Conf. on Plasma Phys., Vol. 31F (2007) p. P4.164.

${ }^{36}$ Y. Peysson, J. Decker, and L. Morini, Plasma Phys. Control. Fusion 54, 045003 (2012). 